\title{
Correlação entre a pressão arterial sistêmica aferida por método oscilométrico com a idade e o nível sérico de sódio em cães hígidos
}

\author{
Correlation between systemic blood pressure measured by \\ oscillometry and age and serum sodium level in healthy dogs
}

\author{
Eduardo Garcia Fontoura ${ }^{1 *}$; Ariana Gayer Ferro ${ }^{2}$; Anelize de Oliveira Campello \\ Felix $^{3}$; Eduardo Negri Mueller ${ }^{4}$; Márcia de Oliveira Nobre ${ }^{5}$
}

\begin{abstract}
Resumo
A pressão arterial vem recebendo destaque na clínica veterinária atual, podendo ser mensurada de forma não-invasiva, pelo método oscilométrico, entre outros. Fatores como a idade já foram relacionados com distúrbios na pressão arterial. O sódio, elemento vital na fisiologia celular de animais, já foi relacionado com o aumento da pressão arterial. Desta forma, este trabalho correlaciona a idade, os valores da pressão arterial sistêmica, sistólica e diastólica, com os níveis séricos de sódio em cães adultos hígidos. Foram estudados 23 cães adultos, hígidos, de ambos os sexos e idade variável, alimentados com ração comercial (standard). As pressões arteriais, sistólica (PAS) e diastólica (PAD), e pulso foram aferidas por esfigmomanômetro digital humano. O nível sérico de sódio foi obtido pelo método de reagente enzimático em modo cinético, utilizando kit comercial. Foi realizada a Correlação de Pearson entre as variáveis. Os valores da PAS, PAD, pulso e sódio sérico, variaram respectivamente de $96-184 \mathrm{mmHg}$, 53- $137 \mathrm{mmHg}, 64-135 \mathrm{bpm}$ e $120-157 \mathrm{mEq} / \mathrm{L}$. Foi observada uma correlação positiva entre as variáveis idade e PAS, idade e PAD, sódio e PAS, e sódio e PAD, enquanto os valores de idade e sódio sérico, demonstraram uma correlação negativa. Nas condições deste estudo conclui-se que há pouca interferência nos valores de pressão arterial, sistólica e diastólica, pela idade e o nível sérico de sódio, em cães adultos hígidos.
\end{abstract}

Palavras-chave: Pressão diastólica, pressão sistólica, pulso, oscilométrico, dosagem de sódio

\begin{abstract}
Blood pressure has received attention in current veterinary clinic and can be measured in a non-invasive way by the oscillometric method, among others. Factors such as age have already been related to disorders on blood pressure. Sodium, vital element in cell physiology of animals, has been reported to increase blood pressure. Thus, this work correlates age, the values of blood pressure, both systolic and diastolic, with the serum levels of sodium in healthy adult dogs. We studied 23 adult healthy dogs of both sexes and varying age fed with commercial feed (standard). The arterial pressure, systolic (SBP) and diastolic (DBP), and pulse were measured by digital human sphygmomanometer. The level of serum sodium was obtained by the method of enzyme reagent in kinetic mode, using a commercial kit. We performed Pearson correlation between variables. The values of SBP, DBP, pulse and serum sodium varied respectively from $96-184 \mathrm{mmHg}, 53-137 \mathrm{mmHg}, 64-135 \mathrm{bpm}$ and $120-157 \mathrm{mEq} / \mathrm{L}$. We
\end{abstract}

\footnotetext{
1 Discente de Mestrado, Programa de Pós-Graduação em Veterinária, Universidade Federal de Pelotas, UFPel, Pelotas, RS. E-mail: eduardogfontoura@gmail.com

2 Discente de Medicina Veterinária, UFPel, Pelotas, RS. E-mail:ariana.gayer@bol.com.br

3 Discente de Doutorado, PPG, Veterinária, UFPel, Pelotas, RS. E-mail: anelizecampellofelix@gmail.com

4 Prof. Dr. do Instituto Federal Catarinense, Campus Concórdia, Concórdia, SC. E-mail: enmuellervet@yahoo.com.br

5 Prof $^{\mathrm{a}} \mathrm{Dr}^{\mathrm{a}}$ do Dept ${ }^{\mathrm{o}}$ de Clínicas Veterinária, UFPel, Pelotas, RS. E-mail: marciaonobre@gmail.com

Autor para correspondência
} 
observed a positive correlation between the variables age and SBP, age and DBP, SBP and sodium, and sodium and DBP, while the values of serum sodium and age showed a negative correlation. Regarding this study conditions, we conclude that there is little interference in the values of blood pressure, both systolic and diastolic, regarding age and serum sodium in healthy adult dogs.

Key words: Diastolic pressure, systolic pressure, pulse, oscillometric, sodium dosage

\section{Introdução}

A hipertensão arterial sistêmica é uma enfermidade que vem ganhando cada vez mais espaço e reconhecimento na prática veterinária (JEPSON et al., 2005). Fatores como a idade, raça, sexo, temperamento, entre outros, já foram descritos como predisponentes a hipertensão arterial. Alterações em parâmetros normais de pressão arterial podem gerar danos em diferentes tecidos, tanto como causa primária como secundária. $\mathrm{O}$ rim pode sofrer danos diretamente nos capilares glomerulares e, consequentemente, diminuir de forma progressiva sua função, reflexos de alterações também podem ser evidenciados no sistema nervoso, cardíaco, endócrino e pulmonar (BODEY; MICHELL, 1997; BROWN; HENIK, 1998; SANTOS; HAIBARA, 2001; BROWN et al., 2007).

O diagnóstico da hipertensão arterial sistêmica pode ser obtido tanto por métodos invasivos (diretos), como por métodos não-invasivos (indiretos). Muito embora o método invasivo seja mais preciso e confiável, este tipo de aferição se torna difícil de utilizar na rotina clínica devido a dificuldades na técnica. Em contra partida, a praticidade e facilidade de utilização fazem dos métodos não-invasivos, como a aferição por método oscilométrico, doppler ou aparelhos de fotopletismografia, uma alternativa de utilização na rotina clínica veterinária, uma vez que valores confiáveis de pressão arterial sistólica podem ser obtidos tanto por meio do método Doppler vascular quanto do método oscilométrico, para animais de pequeno a grande porte, porém valores de pressão arterial diastólica, obtidos pelos dois métodos, não se correlacionam devido variação de seus valores, de forma especialmente marcante nos animais de pequeno e médio porte (PORCIELLO et al., 2004;
CABRAL et al., 2010; PELLEGRINO et al., 2010).

O sódio, elemento vital na fisiologia celular de animais já foi relacionado com o aumento da pressão arterial em pacientes humanos que ingeriam dieta com alto teor do elemento, sendo constatada correlação linear positiva entre a hipertensão arterial com a alta ingestão de sódio (MOLINA et al., 2003), inclusive, de acordo com diferentes etnias (COOPERATIVE RESEARCH GROUP, 1996). Ainda foram descritas associações entre a hipertensão arterial resultando de um defeito na capacidade renal de excretar o sódio, assim como o aumento da ingestão de sódio resultando em uma hipertensão arterial transitória, em diversos modelos experimentais (GUYTON, 1990; FAZAN JUNIOR; SILVA; SALGADO, 2001).

Considerando a importância da relação do sódio com o sistema circulatório, a influência da idade como fator predisponente a alterações, e a necessidade de avaliar a pressão arterial em cães, o objetivo deste estudo foi correlacionar a idade e o nível sérico de sódio, com os valores das pressões arteriais sistêmicas, sistólica e diastólica de cães hígidos.

\section{Material e Métodos}

Para a inclusão no estudo foram considerados cães adultos sem manifestações clínicas, alimentados com ração comercial (standard) e de ambos os sexos. Foram excluídos do estudo animais com histórico de doença recente (seis meses), caquéticos e obesos. Foram utilizados 23 cães entre machos (5) e fêmeas (18), adultos (2 a 11 anos de idade), com média de $15 \mathrm{~kg}$, sem histórico de doença renal e/ou endocrinopatias, oriundos da cidade de Pelotas/RS. Os animais não foram deslocados do seu local de 
origem, e ainda assim foram mantidos em repouso por um período de cinco minutos antes da obtenção dos valores de pressão arterial sistêmica, pulso e coleta de sangue para dosagem de nível sérico de sódio.

Foi utilizado o método não-invasivo oscilométrico, para a aferição das pressões arterial sistêmica, sistólica (PAS), diastólica (PAD), e pulso através do uso de esfigmomanômetro digital humano (Monitor de Pressão Arterial Digital Automático de Pulso, Techline - Z-46. Rossmax International Ltd., Taiwan/China), o qual possui manguito fixo e flexível. Para padronizar a aferição, os cães foram posicionados em decúbito lateral esquerdo, o aparelho foi posicionado sobre a região distal da tíbia direita, e então inflado automaticamente a uma pressão suprassistólica, e em seguida desinflado automaticamente de modo progressivo. Foram realizadas três mensurações entre PAS, PAD e pulso, com um intervalo de 30 segundos e calculada a média entre as aferições. Os valores de PAS e PAD foram agrupados em quatro níveis de acordo com Tilley e Goodwin (2002), sendo normal (PAS 110 - $120 \mathrm{mmHg}$ e PAD $70-80 \mathrm{mmHg}$ ), discretamente elevada (PAS de 120 - 170mmHg e PAD de 80 - 100mmHg), moderadamente elevada (PAS de $170-200 \mathrm{mmHg}$ e PAD de $100-120 \mathrm{mmHg}$ ) e acentuadamente elevada (PAS $>$ de $200 \mathrm{mmHg}$ e $\mathrm{PAD}>120 \mathrm{mmHg}$ ).

Após a obtenção dos dados de pressão arterial sistêmica, foi realizada coleta de sangue para análise dos níveis séricos sódio, através do método de reagente enzimático em modo cinético. O sangue foi coletado de modo asséptico, por punção da veia cefálica, utilizando seringa de $3 \mathrm{~mL}$ acoplada a agulha de calibre $25 \times 07 \mathrm{~mm}$ descartáveis. A amostra sanguínea foi depositada em tubo contendo gel separador com ativador de coágulo (Vacuplast
Collet Line), o qual foi centrifugado (10.000rpm durante 10 minutos) para obtenção do soro. A análise foi realizada em aparelho BioClion Mindray BA88A, seguindo recomendações do fabricante do kit comercial (LabTest - Sódio Enzimático, Ref.: 124). Foram considerados como fisiológicos os valores entre 141,1 e 152mEq/L (BRANDÃO et al., 1999).

Para medir o grau de correlação, entre as variáveis, idade e PAS/PAD, idade e sódio, e sódio e PAS/PAD, foi utilizado o teste de Correlação de Pearson, por meio do programa "STATISTIX 9.0", considerando significativo o valor de $\mathrm{p}<0,05$.

\section{Resultados e Discussão}

A PAS variou de 96 a $184 \mathrm{mmHg}$, enquanto que a PAD de 53 a $137 \mathrm{mmHg}$. Na espécie canina os valores descritos como fisiológicos encontram-se entre 110 a $120 \mathrm{mmHg}$ para a PAS, e PAD de 70 a 80mmHg (TILLEY; GOODWIN, 2002). Dos 23 animais incluídos no estudo, quatro encontraramse abaixo dos valores normais, somente um apresentou valores de PAS considerados como normal, enquanto que 15 encontraram-se na faixa descrita como discretamente elevada e três animais com a PAS moderadamente elevada. Já quando observados os resultados obtidos da PAD, três animais apresentaram valores abaixo do considerado como padrões normais, quatro com PAD normal, seis discretamente elevada, cinco moderadamente elevada e ainda cinco na taxa classificada como acentuadamente elevada (Tabela 1). Neste estudo o pulso variou de 64 a $135 \mathrm{bpm}$, sendo que, do total de animais, 20 animais encontraram-se na faixa caracterizada como fisiológica para cães adultos (70 - 120bpm), sendo que apenas um cão apresentou valores abaixo dos padrões, e três animais acima do considerado como fisiológico. 
Tabela 1. Medidas de pressão arterial sistólica, diastólica, pulso e valores de sódio sérico em cães hígidos adultos alimentados com ração comercial.

\begin{tabular}{|c|c|c|c|c|c|}
\hline Cães (n) & Idade (anos) & PAS (mmHg) & PAD (mmHg) & Pulso & Sódio $(\mathrm{mEq} / \mathrm{L})$ \\
\hline 1 & 2 & 99 & 77 & 79 & 141,0 \\
\hline 2 & 10 & 139 & 88 & 100 & 134,0 \\
\hline 3 & 3 & 184 & 137 & 109 & 140,0 \\
\hline 4 & 3 & 161 & 111 & 64 & 141,0 \\
\hline 5 & 5 & 160 & 120 & 116 & 134,0 \\
\hline 6 & 2 & 96 & 57 & 88 & 149,0 \\
\hline 7 & 3 & 161 & 125 & 90 & 152,0 \\
\hline 8 & 2 & 145 & 86 & 123 & 139,0 \\
\hline 9 & 2 & 108 & 80 & 77 & 120,0 \\
\hline 10 & 3 & 131 & 74 & 93 & 142,0 \\
\hline 11 & 3 & 127 & 95 & 90 & 151,0 \\
\hline 12 & 4 & 179 & 124 & 105 & 131,0 \\
\hline 13 & 6 & 146 & 92 & 94 & 157,0 \\
\hline 14 & 10 & 149 & 101 & 109 & 137,0 \\
\hline 15 & 4 & 145 & 79 & 110 & 139,0 \\
\hline 16 & 2 & 106 & 53 & 135 & 139,0 \\
\hline 17 & 3 & 164 & 131 & 100 & 147,0 \\
\hline 18 & 2 & 137 & 109 & 115 & 138,0 \\
\hline 19 & 11 & 144 & 79 & 90 & 137,0 \\
\hline 20 & 3 & 110 & 69 & 92 & 130,0 \\
\hline 21 & 9 & 150 & 85 & 97 & 135,0 \\
\hline 22 & 7 & 150 & 110 & 125 & 133,0 \\
\hline 23 & 11 & 180 & 136 & 72 & 144,0 \\
\hline Mediana & 3,0 & 145,00 & 137,0 & 97,0 & 139,0 \\
\hline IC Lo & 3,3969 & 131,25 & 85,763 & 91,242 & 136,05 \\
\hline Up & 6,1683 & 153,18 & 107,11 & 106,41 & 143,08 \\
\hline
\end{tabular}

$\mathrm{n}=$ numero de animais; PAS = pressão arterial sistólica; PAD = pressão arterial diastólica; IC = Intervalo de confiança.

Fonte: Elaboração dos autores.

O aparelho utilizado neste estudo gera simultaneamente os valores de PAS, PAD e pulso, conforme apresentados anteriormente, estudos demonstraram que ao serem utilizados os métodos não-invasivos, como o método oscilométrico ou Doppler, os valores de PAS são mais confiáveis (STEPIEN et al., 2003; CABRAL et al., 2010). As variações na pressão arterial podem se manifestar de forma silenciosa, resultando em hipertensão arterial, a qual é classificada em primária, secundária, idiopática, genética, ou também momentânea, esta última, descrita quando desencadeada por excitação ou ansiedade do paciente, os quais resultam numa série de alterações no sistema nervoso autônomo, predispondo a situação de estresse (MISHINA et al., 1997; BROWN; HENIK, 1998; BROWN et al., 2007; SOARES et al., 2012).

Os valores encontrados na análise de sódio sérico variaram de $120-157 \mathrm{mEq} / \mathrm{L}$, sendo que 16 cães apresentaram valores entre $120-141 \mathrm{mEq} / \mathrm{L}$, considerados abaixo dos padrões fisiológicos para a espécie, seis, variando de $142-152 \mathrm{mEq} / \mathrm{L}$, neste caso inclusos na faixa de normalidade, e um cão acima dos padrões, $157 \mathrm{mEq} / \mathrm{L}$.

Quando analisados, os resultados apresentaram correlação positiva de fraca intensidade entre as variáveis idade e PAS $(\mathrm{r}=0,3737, \mathrm{p}=0,0789)$, e idade e PAD ( $r=0,1702, p=0,4373)$, quanto aos valores de idade e sódio sérico, foi observada correlação negativa de ínfima intensidade ( $\mathrm{r}=$ 
$-0,0910, \mathrm{p}=0,6795)$ (Figura 1), e entre os valores positiva de fraca intensidade $(\mathrm{r}=0,1043, \mathrm{p}=0,6355$; de sódio e PAS, e sódio e PAD houve correlação $\quad \mathrm{r}=0,1265, \mathrm{p}=0,5651$ ).

Figura 1. Demonstração da correlação negativa entre a idade e o nível sérico de sódio em cães hígidos alimentados com ração comercial.

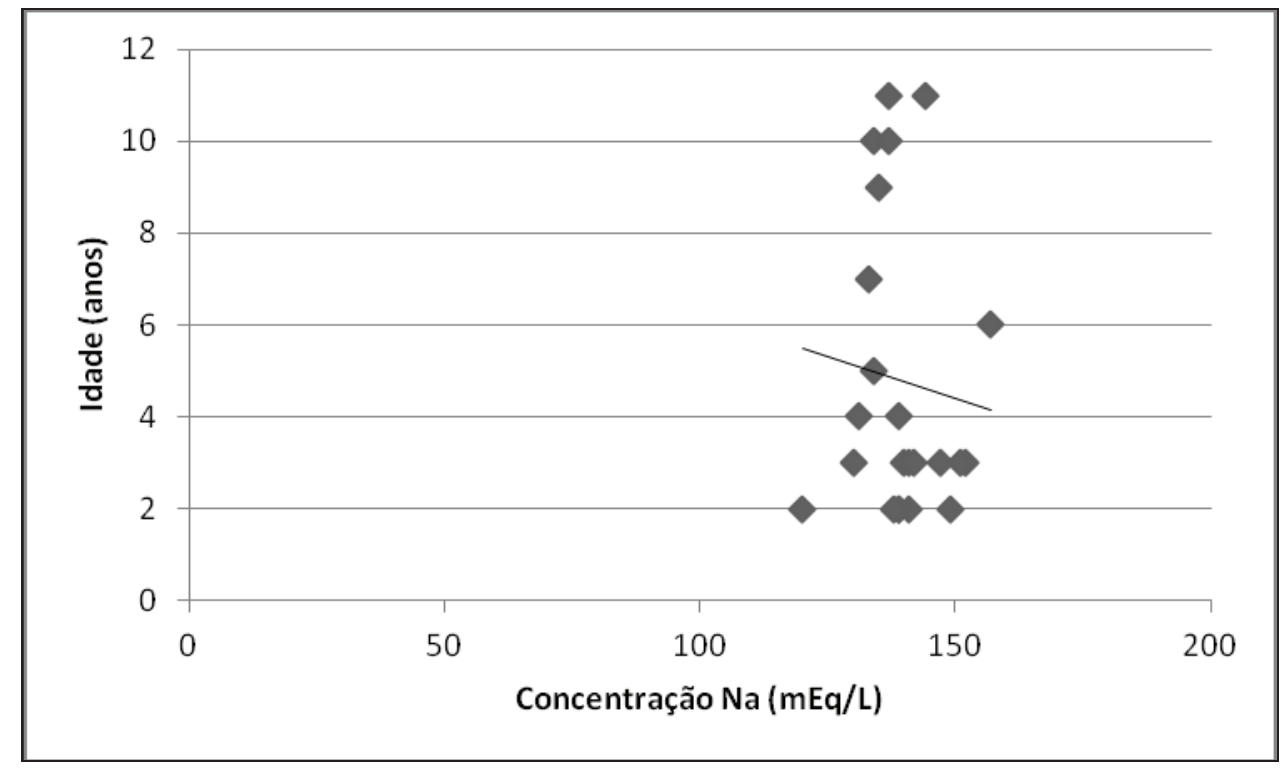

Fonte: Elaboração dos autores.

Alterações na pressão arterial estão relacionadas com manifestações de doenças ligadas principalmente ao sistema cardiovascular, renal e animais obesos. Esta correlação entre pressão arterial e idade, observada neste estudo era esperada, visto que a idade figura entre um dos principais fatores predisponentes a hipertenção arterial (BODEY; MICHELL, 1997; BROWN; HENIK, 1998; SANTOS; HAIBARA, 2001; SOARES et al., 2012). Neste estudo foram considerados apenas cães que recebiam dieta comercial (standard), as quais possuem teores de sódio controlado, desta maneira eliminando a influência da variável dieta sobre os resultados de pressão arterial. Esta influência já foi verificada em humanos e modelos experimentais submetidos a dieta hipersódica, onde esses desenvolveram hipertensão arterial (COOPERATIVE RESEARCH GROUP, 1996; FAZAN JUNIOR; SILVA; SALGADO, 2001).
Nas condições deste estudo conclui-se que há pouca interferência nos valores de pressão arterial, sistólica e diastólica, pela idade e o nível sérico de sódio, em cães adultos hígidos.

\section{Agradecimentos}

Ao CNPq e CAPES, pelas bolsas de pósgraduação e auxílio no desenvolvimento de pesquisa. Beneficiário de auxílio financeiro CAPES - Brasil.

\section{Referências}

BODEY, A. R.; MICHELL, A. R. Longitudinal studies of reproducibility and variability of indirect (oscillometric) blood pressure measurements in dogs: evidence for tracking. Research in Veterinary Science, Oxford, v. 63, n. 1, p. 15-21, 1997. Disponível em: $<$ http://www.sciencedirect.com/science/article/pii/ S0034528897901521>. Acesso em: 19 jun. 2012. 
BRANDÃO, L. P.; HAGIWARA, M. K.; KOGIKA, M. M.; IKESAKI, J. Y. H.; KAWAHARA, R.; WIRTHL, V. A. B. F. Variação dos níveis séricos de sódio, potássio e glicose de cães em choque séptico. Ciência Rural, Santa Maria, v. 29, n. 4, p. 675-679, 1999. Disponível em: <http://www.scielo.br/pdf/cr/v29n4/a18v29n4.pdf>. Acesso em: 02 jul. 2012.

BROWN, S. A.; ATKINS, C.; BAGLEY R.; CARR, A.; COWGILL, L.; DAVIDSON, M.; EGNER, B.; ELLIOTT J.; HENIK, R.; LABATO, M.; LITTMAN, M.; POLZIN, D.; ROSS, L.; SNYDER P.; STEPIEN, R. Guidelines for the identification, valuation, and management of systemic hypertension in dogs and cats. Journal of Veterinary Iinternal Medicine, Lakewood, v. 21, n. 3, p. 542-558, 2007.

BROWN, S. A.; HENIK, R. A. Diagnosis and treatment of systemic hypertension. Veterinary Clinics of North America: Small Animal Practice, Philadelphia, v. 28, n. 6, p. 1481-1494, 1998.

CABRAL, R. R.; CIASCA, B. D.; OLIVEIRA, V. M. C.; VAZ-CURADO, A. P.; LARSSON, M. H. M. A. Valores de pressão arterial em cães pelos métodos oscilométrico e Doppler vascular. Arquivo Brasileiro de Medicina Veterinária e Zootecnia, São Paulo, v. 62, n. 1, p. 64-71, 2010. Disponível em: <http://www.scielo.br/pdf/abmvz/ v62n1/v62n1a09.pdf>. Acesso em: 12 jun. 2012.

FAZAN JUNIOR, R.; SILVA, V. J. D.; SALGADO, H. C. Modelos de hipertensão arterial. Revista Brasileira de Hipertensão, São Paulo, v. 8, p. 19-29, 2001. Disponível em: <http://departamentos.cardiol.br/dha/ revista/8-1/004.pdf>. Acesso em: 12 jun. 2012.

GUYTON, A. C. Long-term arterial pressure control: an analysis from animal experiments and computer and graphic models. American Journal of Physiology, Bethesda, v. 259, n. 5, p. 865-877, 1990.

INTERSALT COOPERATIVE RESEARCH GROUP. An international study of electrolyte excretion and blood pressure: results for 24 hour urinary sodium and potassium excretion. BMJ, London, v. 297, n. 1249, p. 319-328, 1996. Disponível em: <http:/www.bmj.com/ content/312/7041/1249>. Acesso em: 17 jun. 2012.

JEPSON, R. E.; HARTLEY, V.; MENDL, M.; CANEY, S. M.; GOULD, D. J. A comparison of CAT Doppler and oscillometric memoprint machines for non-invasive blood pressure measurement in conscious cats. Journal of Feline Medicine and Surgery, Bristol, v. 7, n. 3, p. 147$152,2005$.
MISHINA, M.; WATANABE, T.; FUJI, K.; MAEDA, H.; WAKAO, Y.; TAKAHASHI, M. A. A clinical evaluation of blood pressure through non-invasive measument using the oscillometric procedure in conscious dogs. Journal of Veterinary Medicine Science, Lakewood, v. 59, n. 11, p. 989-993, 1997.

MOLINA, M. C. B.; CUNHA, R. S.; HERKENHOFF, L. F.; MILL, J. G. Hipertensão arterial e consumo de sal em população urbana. Revista de Saúde Pública, São Paulo, v. 37, n. 6, p. 743-750, 2003.

PELlEGRINO, A.; PETRUS, L. C.; YAMAKI, F. L.; SANTOS, A. L. F.; LARSSON, M. H. M. A. Valores de pressão arterial de cães da raça Golden Retriever clinicamente sadios. Brazilian Journal of Veterinary Research Animal Science, São Paulo, v. 47, n. 4, p. 307-314, 2010. Disponível em: <http:// www.revistasusp.sibi.usp.br/scielo.php?pid=S1413$95962010000400008 \&$ script $=$ sci_arttext $>$. Acesso em: 03 jul. 2012.

PORCIELLO, F.; BIRETTONI, F.; CCONTI, M. B.; MARINETTI, C.; ANTOGNONI, M. T.; FRUGANTI, G. Blood pressure measurements in dogs and horses using the oscillometric technique: personal observations. Veterinary Research Communications, New York, v. 28, n. 4, p. 367-369, 2004. Disponível em: <http://www. springerlink.com/content/uu141188m6317484/>. Acesso em: 10 jun. 2012.

SANTOS, M. J. C.; HAIBARA, A. S. Reflexos cardiovasculares e hipertensão arterial. Revista Brasileira de Hipertensão, Rio de Janeiro, v. 8, n. 1, p. 30-40, 2001.

SOARES, F. A. C.; NEUWALD, E. B.; MOMBACH, V. S.; D'AVILA, A. E. R.; CONRADO, F. O.; GONZÁLEZ, F. H. D. Systolic blood pressure of dogs at hospital and domestic environment. Ciência Rural, Santa Maria, v. 42, n. 7, p. 1243-1248, 2012. Available at:<http://www.scielo.br/scielo.php?pid=S0103$84782012000700017 \&$ script $=$ sci_arttext $>$. Accessed at: 14 jun. 2012.

STEPIEN, L. R.; RAPOPORT, G. S.; HENIK, R. A.; WENHOLZ, L.; THOMAS, C. B. Comparative Diagnostic test characteristics of Oscillometric and doppler ultrasonographic methods in the detection of systolic hypertension in dogs. Journal of Veterinary Internal Medicine, Madison, v. 17, n. 1, p. 65-72, 2003.

TILLEY, L. P.; GOODWIN, J. K. Manual of canine and feline cardiology. In: LARRY, P.; TILLEY, F. W. K.; SMITH JUNIOR, M. A.; OYAMA, M. M. S. Manual of canine and feline cardiology. 3. ed. Philadelphia: W.B. Saunders, p. 337-344, 2002. 